\title{
RaIlWAy SAFETY PRotection WiTH ANDROID MOBILE APPLICATION FOR 5G NEW RADIO NETWORK
}

\author{
Tony Tsang and Man Cheng Chong \\ Centre of International Education, Hong Kong College of Technology, \\ Hong Kong
}

\begin{abstract}
In every night of non-traffic hours, different jobs are conducting maintenance works in "Railway" trackside area. This project will explain a specific section of track under the sole control an Engineer's Person-in-Charge as procedures. And how to provide protection methods by which a person or persons on or near a track are safeguarded from potential train movements or a train is safeguarded from other train movements or obstructions, or persons or equipment are safeguarded from traction power.Consolidated past several investigation reports and according to related is rules, workflow or procedures etc. to summarize. There are protection tools left on trackside area incident caused by the workers are forgetting and poor management. Proposed are different project themes in the light of their expertise, experience and observation in their daily works. The proposed themes are compared, assessed and prioritized under the criteria - "Manageable", "Measurable", "Result of Benefit", "Standardization" and "Priority" in the Decision Matrix. Establish some solve problem methods for comparing to find out which that lower-cost plan accordingly. I came up with a conclusion and the ideas as develop a mobile application and create a unique $Q R$ code label with equipment naming to facilitate each worker management of protection tools. This is also fulfilled in popular terms of Creativity and Innovations. Used the MIT App Inventor (Massachusetts Institute of technology) an intuitive and visual programming preform for mobile application are development. Stage 1: program for individual mobile user application. Stage 2: build-up Network centralized storage with supervising console operation. Stage 3: testing system under with $5 G$ network compatibility, bandwidth and network speed is applicable people will be able to use more of the network dedicated to each mobile phone.Finally, successful to apply trial works a fruitful outcome after implementation of the project solution. There was no missing of protection tools on trackside area within the trial period. With the safety-first culture boosted by us, I believe we can achieve a common goal: Everyone Going Home Safe and Well Every day.
\end{abstract}

\section{KEYWORDS}

Railway Trackside Safety, QR code, Network Centralized Storage, 5G Mobile Network,

\section{INTRODUCTION}

"Railway" has been developed very important in city of main public transport service. A safe, reliable and efficient railway system is paramount importance. Regarding are railway operations are very busy. Committed high standard maintenance works are able to provide safety, reliability and excellent service. The Traction power system, Signalling system and Rail permanent way system etc. must be maintaining relevant regular maintenance works on trackside area in every night of non-traffic hours. As controllers in railway operations, managing track access safely is an absolute prerequisite. One of our initiatives is to ensure protection on track is properly removed on completion of work. When working on track, proper 
protection arrangement must be in places, such as placing Red Flashing Light and Earthing Rod. And when the work is finished, it is important that the working party must remove all of the protection arrangement that is applied by them and at the same time, must not remove or interfare the protection arrangement of other working parties. Safe running of trains after maintenance works must be ensured all tools were cleared in the trackside area. However, some protection tools left on track incident become more than frequently. Therefore, the first passenger train was out of service hit by some about delays, with knock-on effect on road traffic. There's really not solved this problem. You're going to lose money, brand-mark and reputation. Us did not even realize it the losses could take several different formats.

Despite launching propaganda and promotion to drive safety-first culture, it was disappointing that Red Flashing Light left on track incidents still happened occasionally. In a time of rapid growth, we should explore how to further improve the situation. In view of the popularity of mobile application technology, we proactively designed a mobile application for recording the application and removal of protection arrangement. With minimal extra workload to the working parties, the mobile apps can facilitate the checking of proper removal of protection arrangement by the work parties themselves as well as by the station staff.

The project solution achieves the goal: managing safety in a systematic, proactive and consistent manner by mitigating risks contributed by human factors. To turn the initiative into reality, trial was conducted in front line staff and some stations staff with positive feedback. From the success of the trial, we believe that the project solution can be standardized and applied to all lines and all locations.

\section{Methodology AND TeChNOLOGY GUIDE}

\subsection{What is QR Code}

QR comes from the abbreviation of "Quick Response" in English. QR code is a twodimensional bar code used on mobile phone and designated scanner. It is a matrix block and white dot or bar graphic. In addition to representing text, graphics, sound and other information and have large capacity, reliability, confidentiality and anti-counterfeiting of data etc. Meanwhile, this matrix barcode could be stored biggest digits data or Chinese characters and also including pictures with audios, etc. Any material surface is not limited to printing with it is more fault-tolerant to read information completely when QR code slightly damaged. [1]

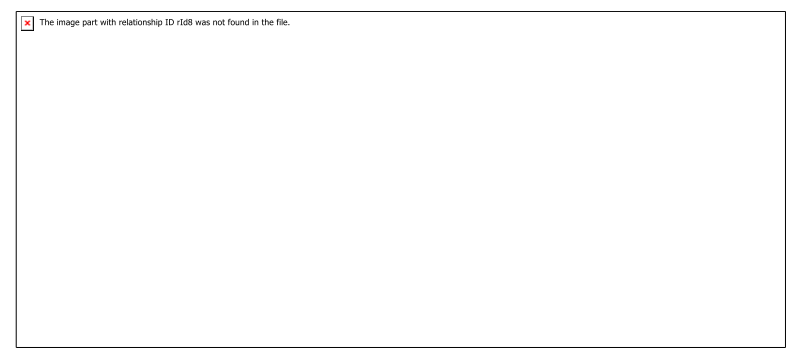

Photo by http://www.pooito.edu.hk/ 


\subsection{MIT APP Inventor}

MIT APP Inventor is an easy to use visual programming platform that allows everyone persons and any stage developers to build up dedicated apps for mobile phones and tablets. Due to the MIT APP Inventor based on blocks-bases tool facilitates to creation. As each apps are programming to running in less than 30 minutes by MIT APP Inventor introduction. So have a simple study, develop and start-up application in a smartphone device. And what's more, diverse blocks-based tool components to create of complex, high-practical apps significantly less time than traditional coded programming environments. The MIT APP Inventor project team seek to software development by authorising all people, to change from technology consumption to innovation. [2]

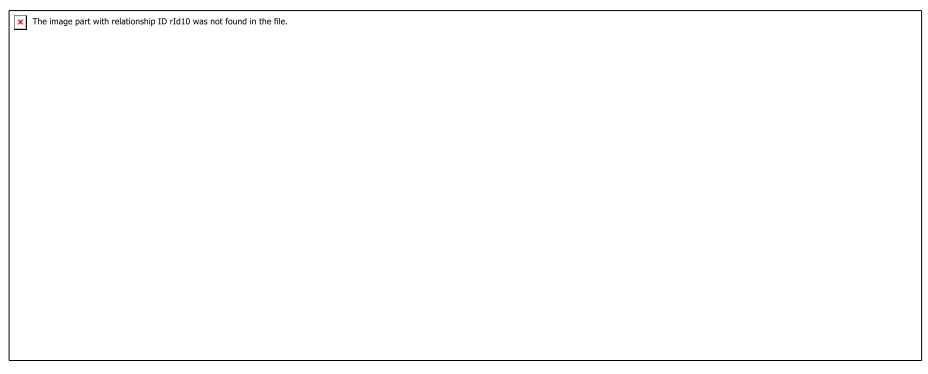

Photo by https://appinventor.mit.edu/

\subsection{Setting APP Inventor}

Layout page will automatically open after Project name is created (Fig.4). Project name in top left corner of the screen. Left is the (Palette). Middle is the (Viewer) and Right is the (Components area) where a user was selected or placed components (Fig.5) [3].

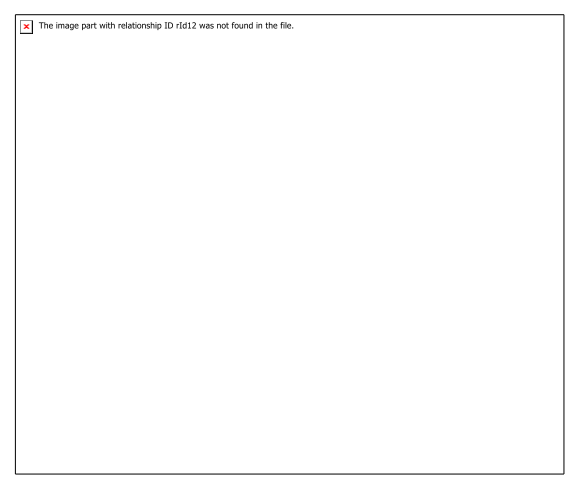

Figure 4

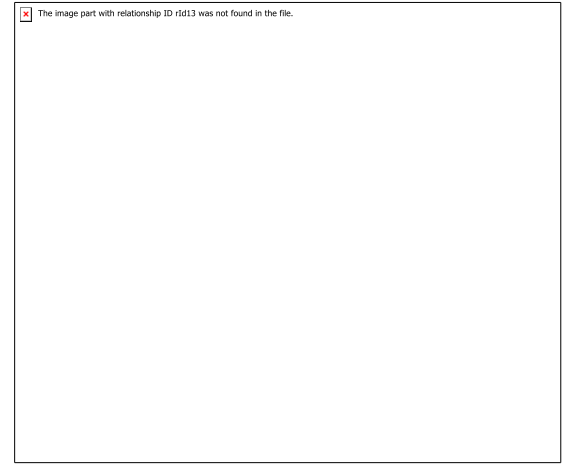

Figure 5

Simple of creating components in App Inventor. Just drag anything from the component area to interface design area. For example, will create a Label component: Drag the Label icon to mobile interface design area. System could be create different named for recognized a lot of components. This project will used components as follow:

\section{$\underline{\text { User interface components [2] }}$}

Label: display text 
Button: the main of the application's interaction with the user. When user pressed the button, program will be executed to achieve the purpose of interaction.

Text Box: let user enter text and the entered text is stored in the property. The text box is usually used in conjection with the Button. Allow the user to press the button for subsequent proceeing after entering text.

Notifer: during execting of the application. It is display some messages to user of the necessary information. Notification box can automatically disappear after displaying message without interfering user's operation. Message can also be displayed in a pop-up dialog box.

\section{Layout components}

Very important in interface design of the application. Despite powerful apps but not popular with users if they have a crude or incompatible interface.

\section{Media components}

Player: make the phone vibration and you can set the vibration time. The Player component in Media category and non-visual component.

\section{$\underline{\text { Sensors components }}$}

Barcorde Scanner: recognizing QR code label.

\section{$\underline{\text { Storage }}$}

TinyDB: database can be used to store data for further processing and analysis in the future.

(Difference of File and TinyDB) File: in the simulator was performed on the simulator or real machine. Data of File will be cleared every time. Execution will start form the empty database. TinyDB: TinyDB database provided by App Inventor 2 has been improved to share. So, performing the simulator or real machine does not clear the data of TinyDB.[4]

Can be displayed on the screen as "visual components". Some components are not displayed when they are executed. It should be under "Blocks" program to operation them such are called "non-visual components". When dragging a non-visual component to interface desihn area during in design plase. Will be placed at the icon in invisible area for indicating that nor be displayed (Fig.7). 


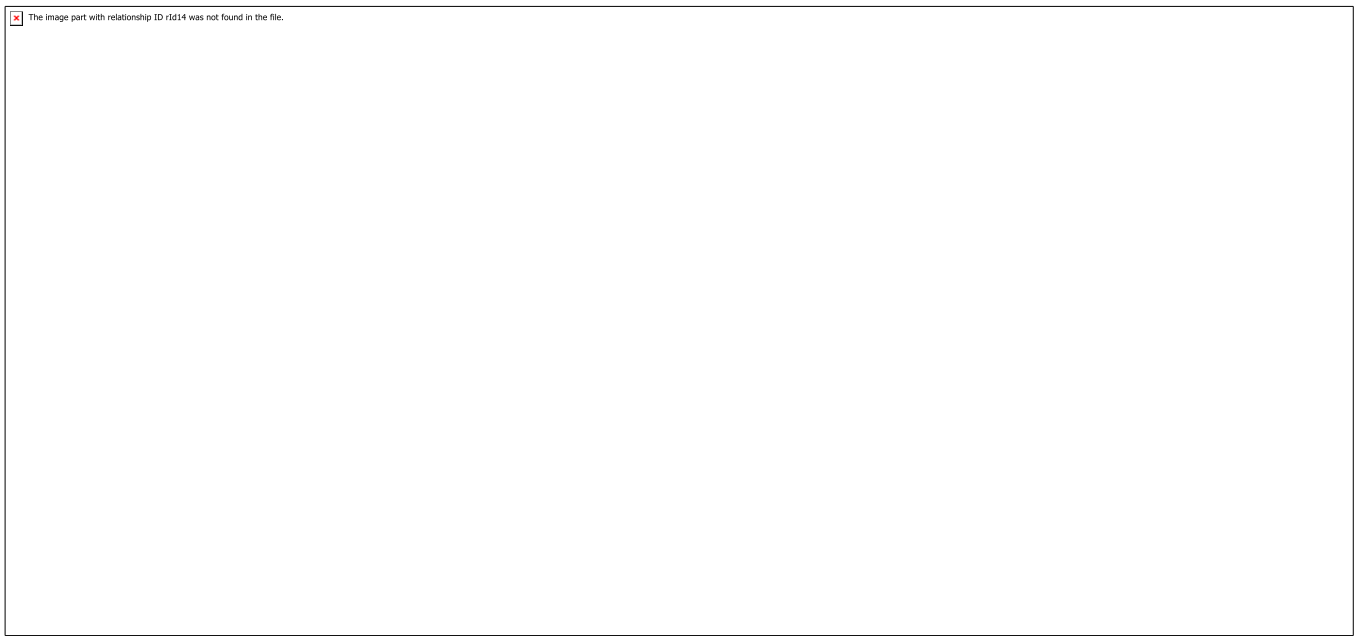

\subsection{Block Function}

Figure 7

When user makes certain instruction in the interface area, such as pressing a button or entering text in a Text Box. Will corresponding event be triggered. While executing control in the program block set by apps designer. The build-in shown on the left side block editing page contains all system's built-in program blocks. About area will corresponding and automatically icon generative under was selected components by designer. When user is selecting any item, system will list all function block for selection. Events, methods and properties as separate different type colours for user recognized easily. How to distinguish different function blocks: Yellow is the Event, Purple is method, Light green is attribute value, Dark green is set attribute value (Fig.8) [2].

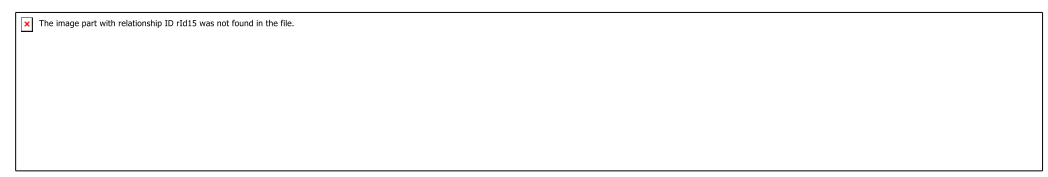

Figure 8

\subsection{Programming}

\subsubsection{Resource Occupation}

In oriented programming patterns, "Block" combination is core of program flow necessary. Designer sets up a situation for which user's to operate in advance. When the application failed to operation, will execute a specific code in response. I have set-up infinite "Loop" function and check at interval time for system to analysis. This method consumes a lot of system resource and it also difficult to set the clock time (if interval is too short, consumes more than system resources; if interval is too long, impossible to respond immediately.)

\subsubsection{Solve the Resource Occupation}

How to solve the problem? Answer is "Event", processing method is "turn active to passive". for example: when system does not actively check whether the button is pressed. So than resources are not wasted. When button is pressed, system is notified by the pop-up prompted 
title: "Transaction No." with shown Text Dalog box for user text input. Subsequent, open another screen with start value after user was input transaction number in Text Dalog box (Fig. 9) [4].

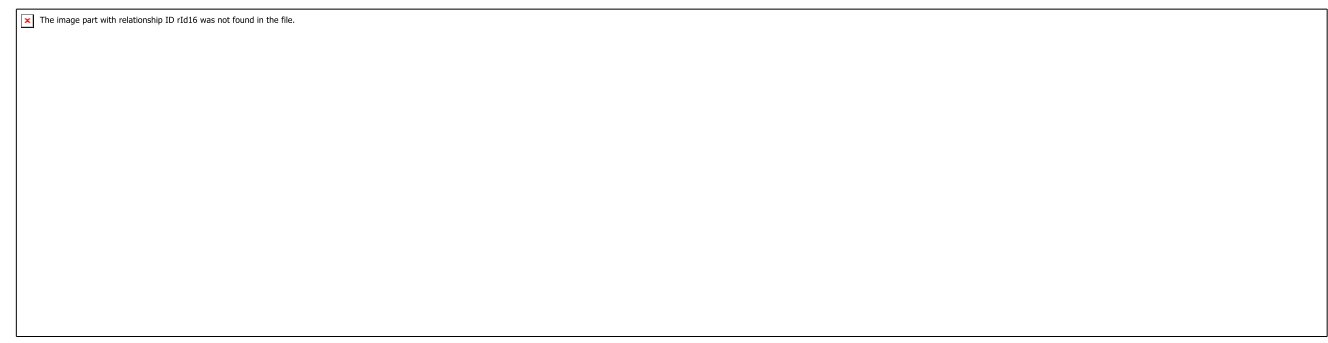

Figure 9

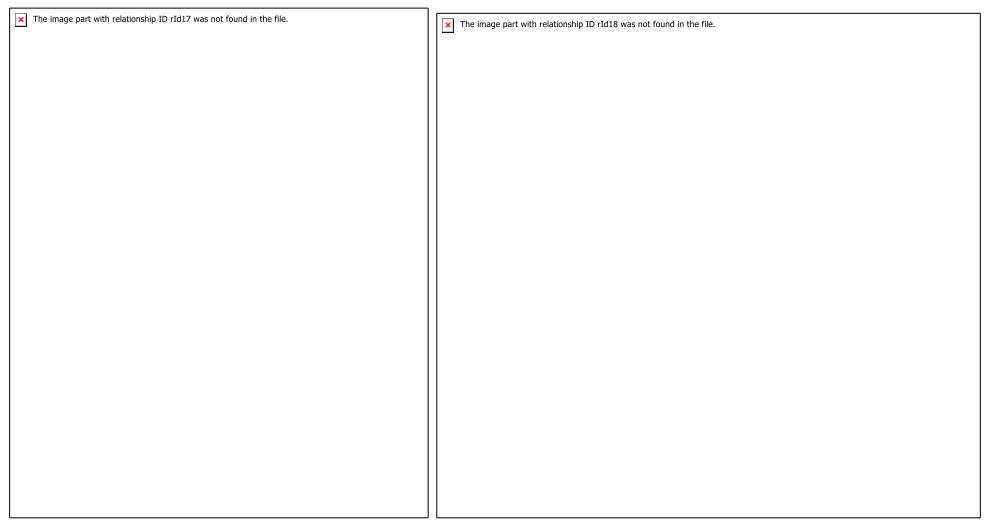

Usually events consist as follow:

1. Event source: the component that triggered the event, such as Button, Text Box, etc.

2. Event Name: the event that occurred, such as Click, Got Focus, etc.

3. Processing code: A piece of program that is executed after the event.

\subsubsection{Access variable value}

There are two ways to access variable value in the program: first method is to move the mouse over the declared variable name. after tile to get and set the variable will be displayed. Click the mouse on the tile Lift click add tile to edit area. Second method is seclect Variable in Built-in item. Then click Get or Set tile and then click variable to be used in drop-down meun to edit area (Fig. 10) [5]. 


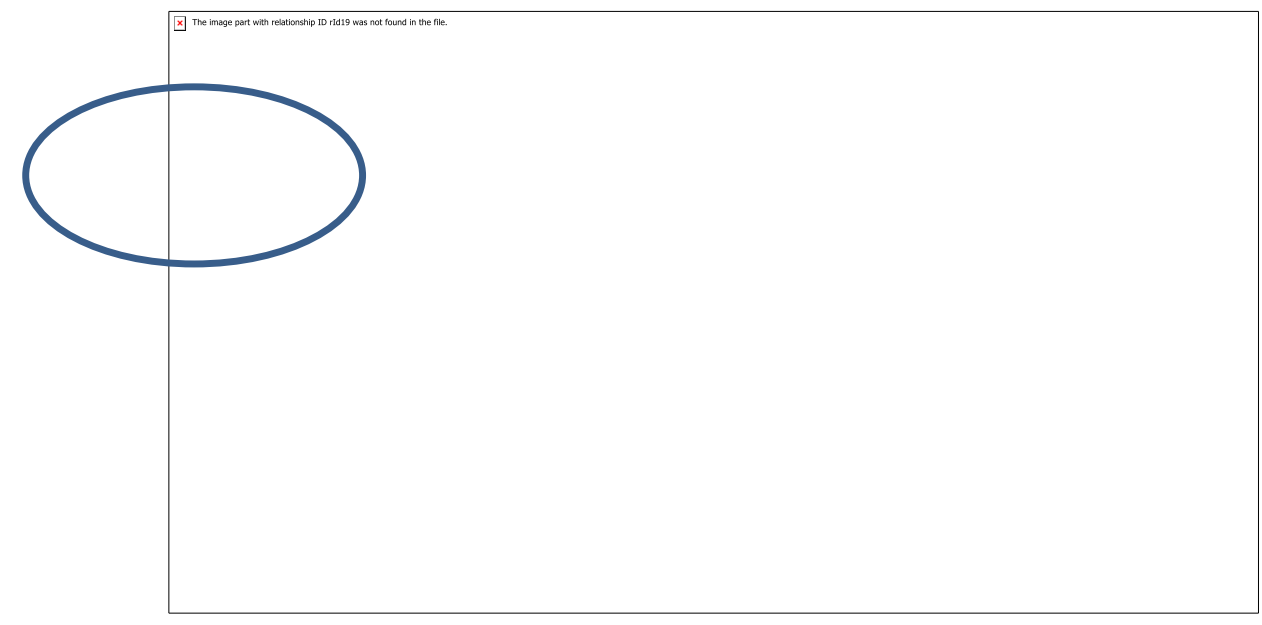

Figure 10

\subsection{Conditional Expression}

Program is usually executed is sequentially. That is according to code by code or one by one step. But sometimes different code need to be complexity situations. The principle is based on conditional expression. Despite the operations are usually used as Comparisons or Logical for calculation. however, both formula such as return True or False.

Comparison operation compares two different items. If compare is correct, it return is true. If compare is incorrect, it return is false. Designer as according different process to a comparison result (Fig. 11).

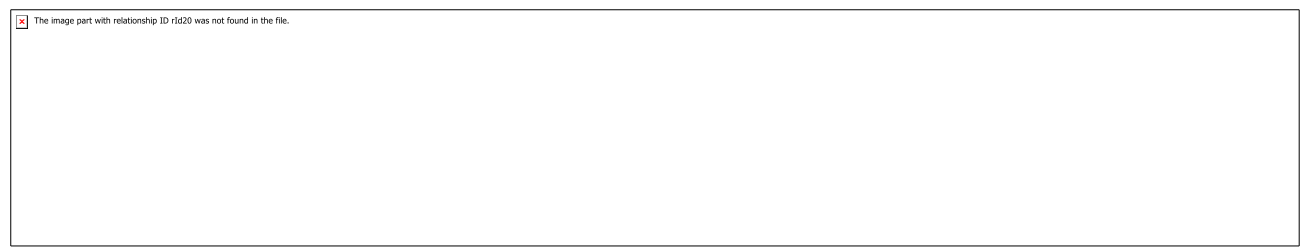

Figure 11

Logiccal operation is combination of multiple comparison to obtain the final result. Which is usually used in more complex situations. There are 4 pieces as "Not, And, Or, Equal" (Fig. 12) [5].

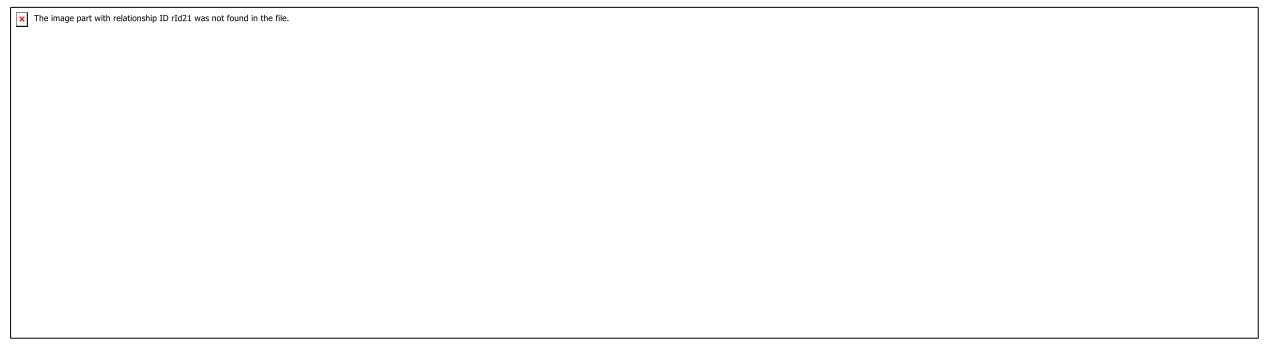

Figure 12 


\subsection{If-Then-Else Expressions}

If-Then: Check the specified conditional expression. When the conditional shown is true, program block in formula will be executed. If opposite, the one-way block will ended immediately.

If-Then-Else: one-way block function is imperfection. Multiple-way block is created by expanding "Else" icon in the one-way tile. (Fig.13,14) [5]

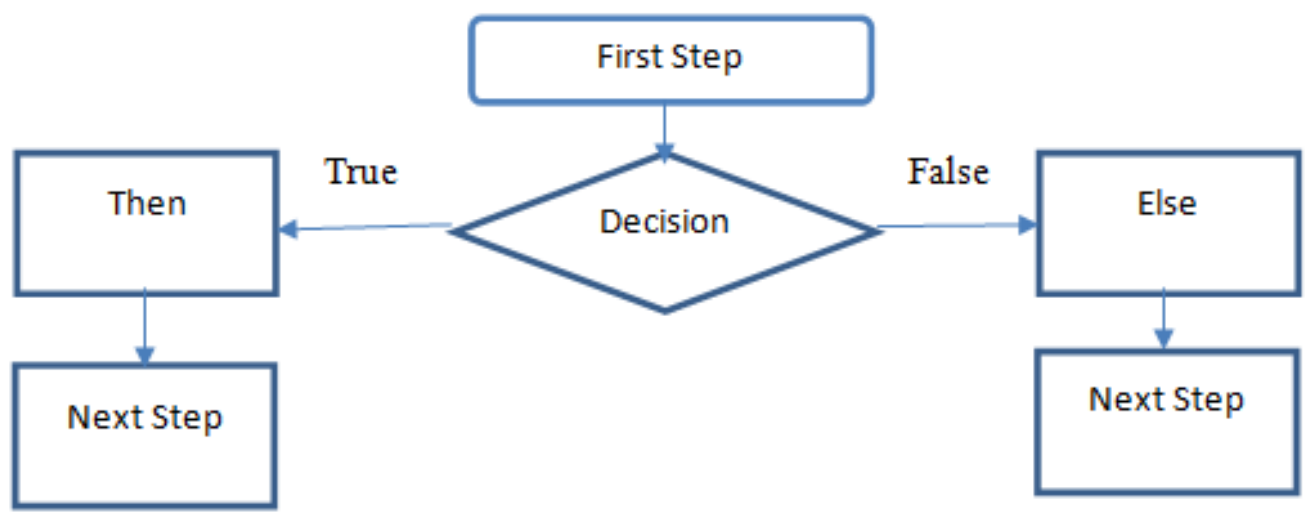

Figure 13

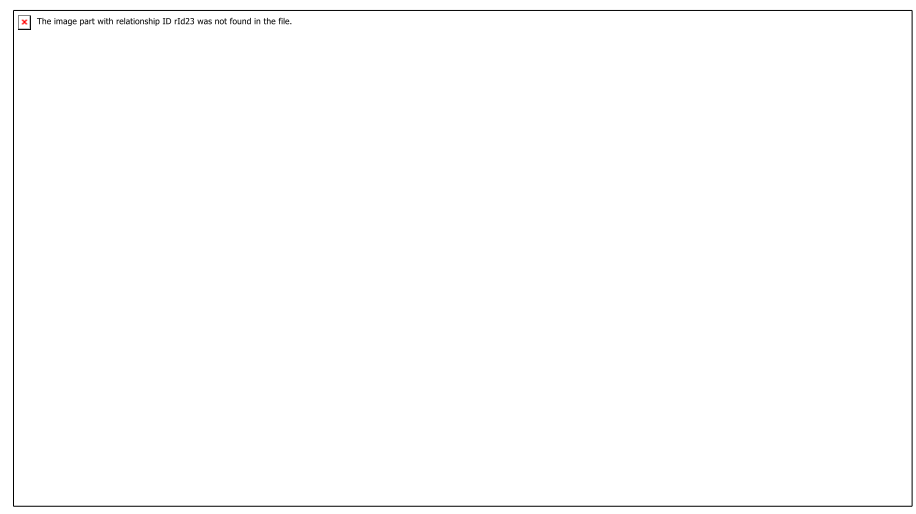

Figure 14

\subsection{Database}

Used database to store data that it can be further processed and analyzed such as more advanced for the application. App Inventor 2 provided TinyDB for storages data in simulator and smartphone. Please note that only provide APK file installation could be used build-in projectspecific TinyDB database for each project. If start up on emulator or a device built-in emulator, it should be temporary stored in shared file. (Fig.15,16) [5, page 234]. 


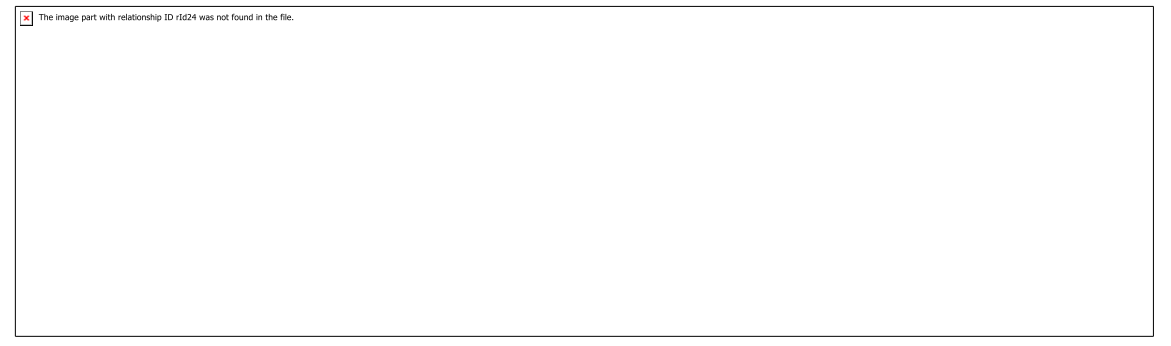

Figure 15

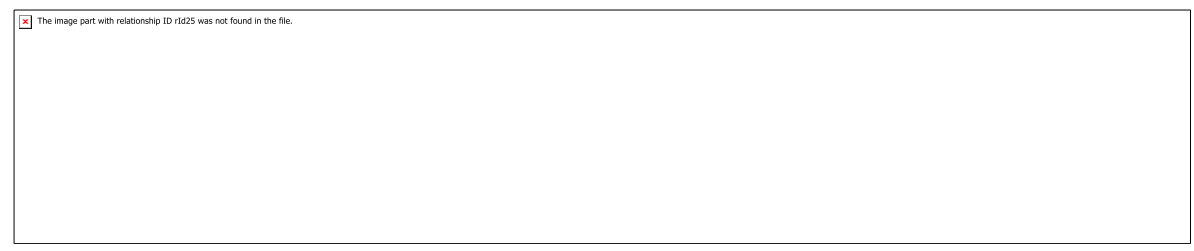

Figure 16: TinyDB as non-visible components

\subsection{Network Database Storge}

FirebaseDB - undoubtedly become the backend as a service (BaaS) platform for app developers. FiredaseDB is a simultaneous support to Android, IOS and webpage with cloud development platform. Provide real-time data synchronized database in server, it can be effective to reduce developing time and assist developers to reinforce in front-end optimization. Main core and three categoies of functions:

1. Firebase Analytics: powerful unlimited data analysis function is the core of products. It have currently free to use with 500 types unlimited records, also setup the customize key event and user attributes. The visual dashboard allow users to monitoring all the important user behaviors and performance analysis of marketing strategy.

2. Develop: provide safe and effective back-end service including is Realtime database, Authentication, Cloud Messaging, Hosting, Strage and Remote configuration. Test Lab for Android and Crash Reporting that functions let developers easily to test app in diffierent operation system with under hardware performance. Quickly found any problem and keep systam operation normally.

3. Grow: easy to set-up message notifications, App Indexing, Dynamic Links and Email Invites function. Integrated of Google AdWords app behavior data, allowing app developers to provide more then accurate advertising or communication messages to target customer.

4. Earn: consolidated with self product's AdMod can join advertising network in easily place to slots. As well as diversified advertising formats with tracking mechanisms. [6] 
International Journal of Computer Science \& Information Technology (IJCSIT) Vol 12, No 4, August 2020

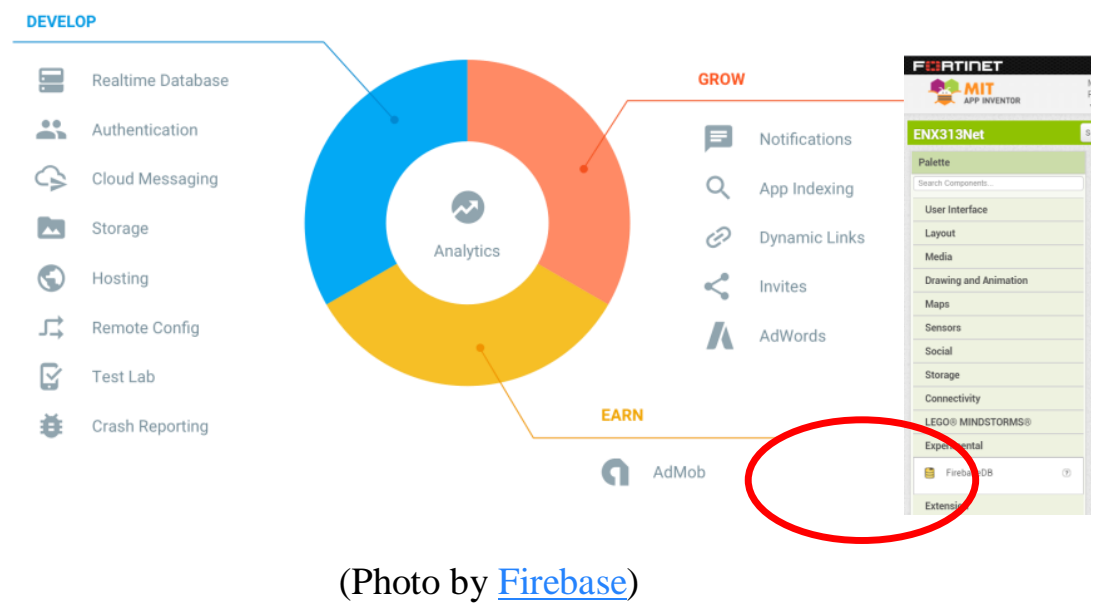

Set-up the component attribute parameter is enx313-2019 to be label used in Realtime Database.

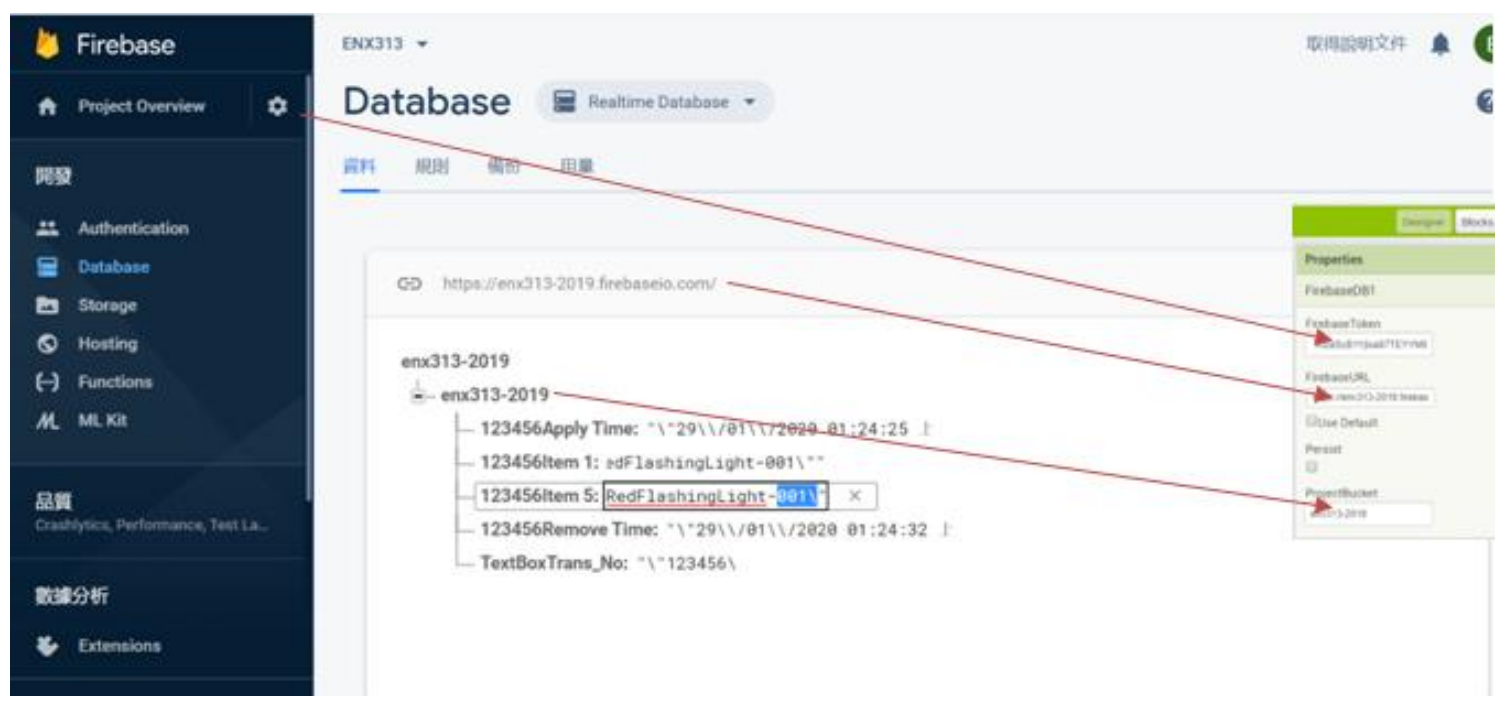

Three categories must be corresponding with App Inventor in FirebaseDB properties:

1. Firebase Token: Authentication credentials are used to identify all users that is an application or website.

2. Firebase URL: Uniform Resource Locator is unique and there will not be two. So that it connected between with each mobile device for communication.

3. Project Bucket: tags your application value will be stored of folder. [7]

Network flow monitoring: automatically measure the network capacity in characteristics of your apps. About data collected with user's behaviours and methods that can understand where and when the performance of mobile apps improvement. (Fig.17) [8] 


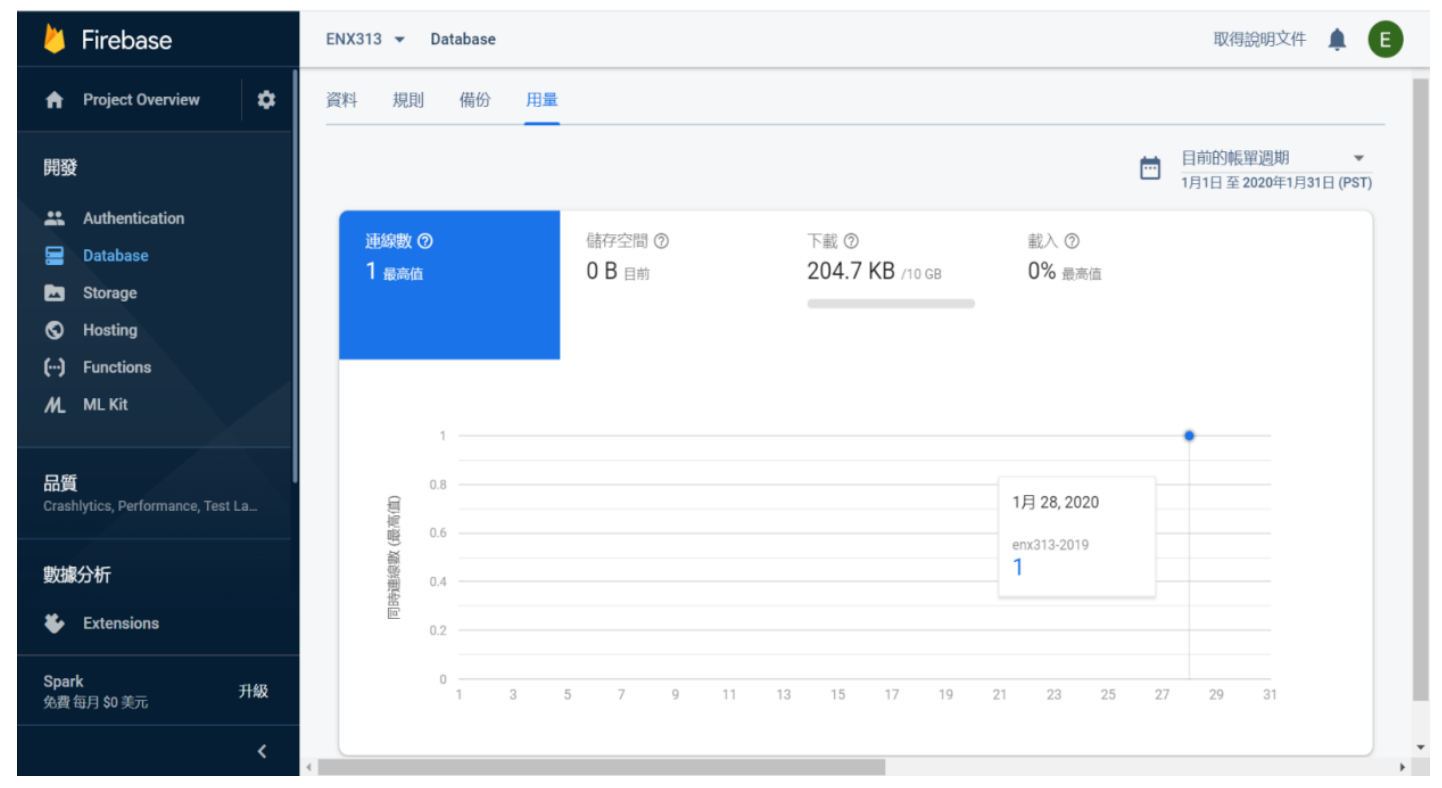

Figure 17

\subsection{Mobile Application Testing and Detect Founding}

Users can create or retrieve a record in the mobile apps by inputting the transaction number or work order of their work. Under each record, users can register the application and removal of several items for a work. Each item is identified by a coding plate with a per-set unique QR code (QR code can be generated in web page: https://www.qr-code-generator.com/ which is to be scanned by the mobile phone through the mobile apps for record. [1]

(1) Press \{Transaction No.\} (2) Press $[\mathrm{OK}]$ after number inputted (3) Show scan button after item selected

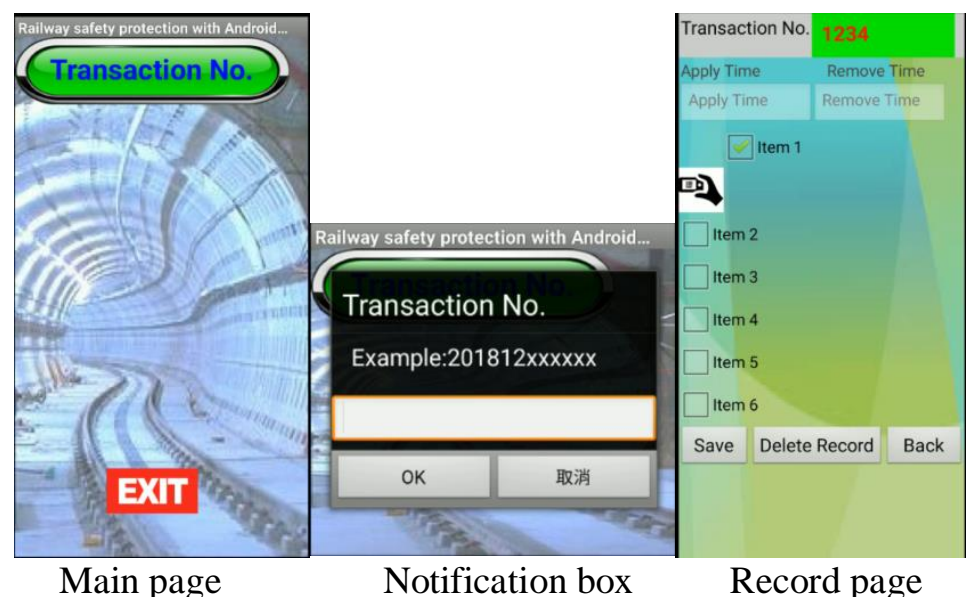
(4) QR code scanning
(5) unique QR code
(6) Incorrect item
(7) Correct item scan 


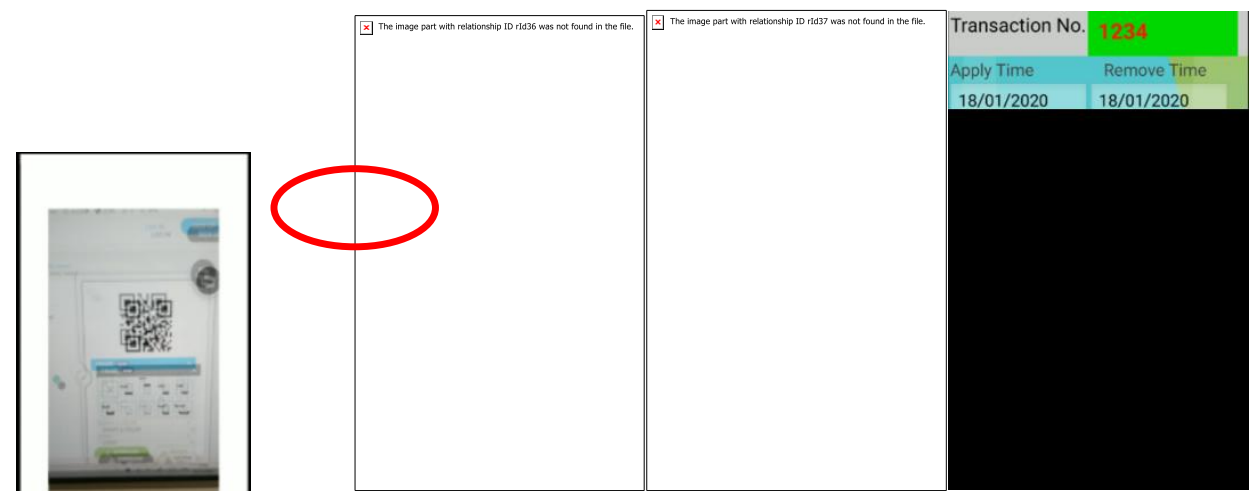

In this trial processing, I found some defective will affect to test result as below:

1. Un-charged battery for the mobile phone.

2. Standard QR nameplate for equipment were not fixed in surface.

3. in dark environment to scanned QR code.

\subsection{Network Monitoring}

Firebase with App Inventor is archiving between System Controllers to users both side communications. The system controller can be comprehensive to monitoring all place in trackside equipment. According the Firebase data records to identify protection item was removed. (Fig. 18)

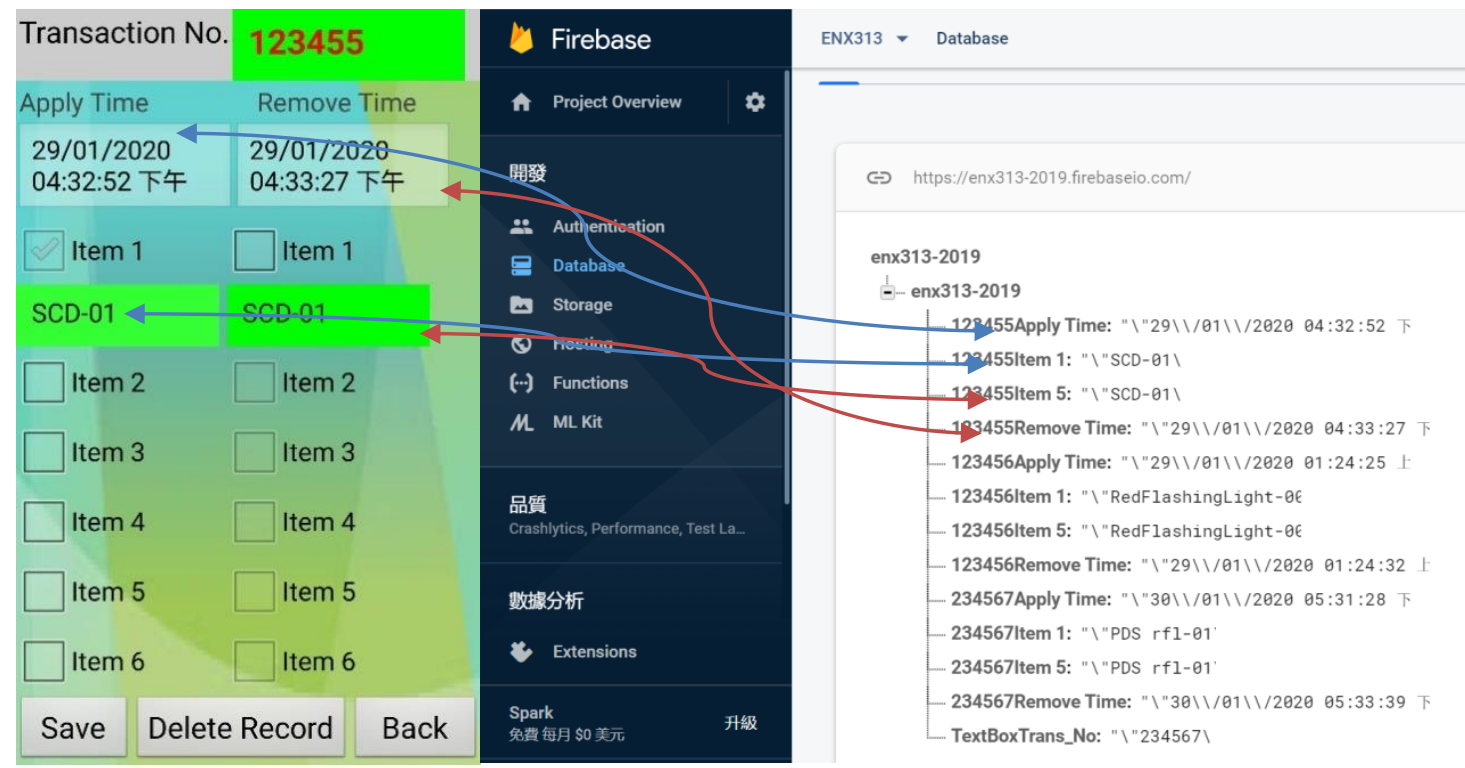

Figure 18

\subsection{Supervision Result}

As the above network supervision result, I would be ensured that communicate between Firebase with Apps Inventor are synchroniy it should have successfully. The Competent Person (CP) / Engineering Possession In-Charge (EPIC) before access to track side. Who will be 
inform with System Controller correctly activaties after conducting anything protection equipment place, apply or remove about correctly behaviors. System controller accordance the network result to keep close monitoring. Meanwhile, it could be summarize, analysis and statistics various workship is periodic after when collected relevant data in server.

\subsection{G Network}

The newly fifth-generation (5G) mobile service $[11,12]$ spectrum released plan for 2020-2022 was published in January 2020 by Communications Authority of Hong Kong. Will release a maximum value of $160 \mathrm{MHz}$ in the $600 / 700 \mathrm{MHz}$ bands and $80 \mathrm{MHz}$ in the $4.9 \mathrm{GHz}$ bands to market in 2021. Simultaneously continue to supply more than spectrum and facilitate public mobility development of service. Besides, $2900 \mathrm{MHz}$ spectrum is available for assignment in $26 / 28 \mathrm{GHz}$ bands. Recently, some industry of mobility companies introduced more next series in which one model 5G mobile phone should be applicable network in Hong Kong. [9]

Although the international have not been definition for 5G. It can roughly cover as two mainstream frequency bands. Sub $6 \mathrm{GHz}$ (that is below $6 \mathrm{GHz}$ ) which no different from the $4 \mathrm{G}$ LTE frequency band have currently to use. Another is up $24 \mathrm{GHz}$ above is "millimeter wave" frequency band that is often heard recently. The 5G network are combines in existing $4 \mathrm{G} \mathrm{LTE}$ frequency band and with a heterogeneous network (HetNet). Through the main speed of millimeter wave (mmWave) broadband technology and with low-power, wide-covering (sub $6 \mathrm{GHz}$ narrow-band technology). It provides most suitable wireless network in different environments to meet the short/long distance Communication requirements. Also, concepts of $5 \mathrm{G}$ included as follow:

Small cell: smaller cell equivalent to coverage of $4 \mathrm{G}$ LTE. The electromagnetic wave's diffraction ability becomes stronger.

Multiple-Input Multiple-Output (Massive MIMO): more transmitter with more receiver for maintains faster speed data transmitting.

Beamforming: the technology of directional signals converts to existing coverage into precise. Through the superposition of transmitting or receiving in a specific directional device. Which not only extends transmission distance but also significantly reduce signal interference. [10]

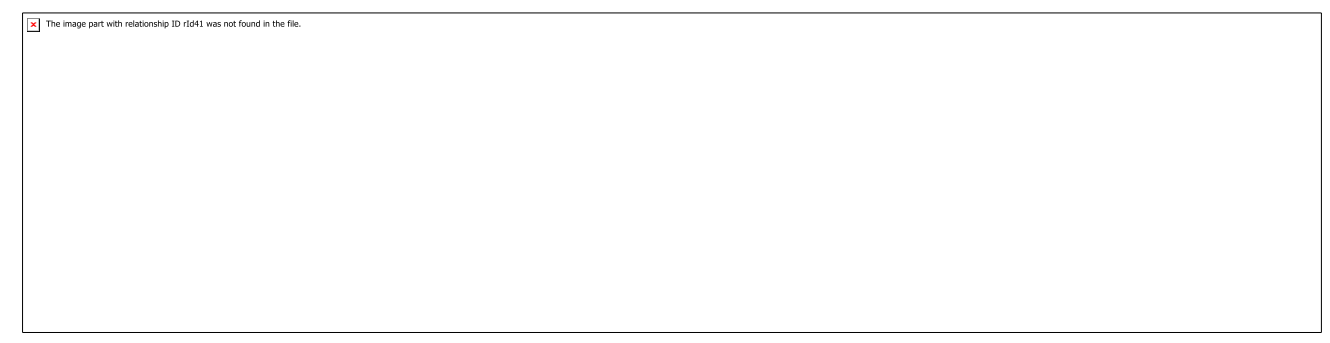

( Photo by [10])

Against at railway situation, track area including tunnel, over ground, viaduct, bridge and remote hinterland area. Must be use stable with reliable communication system to enhance our coordination work. Therefore, the 5G network such as very well with this development system. 


\section{Conclusions}

\subsection{Conclusions}

In this project earlier, I was proposed two-stage as phase one mobile apps development with phase two are cloud network storage. However, in accordance with my Project supervisor's, Dr Tony Tsang from Hong Kong College of Technology is more than discussion, study and experience sharing. He has suggested that the project had including new mobile network technology to rise up performance and reliability. Furthermore, he has reminded me the cloud storage data is part of big data information. It could make the most of data to modify my application while is user's incorrect operation. I very appreciated his professional and ideas.

Due to me unfamiliar with system development logic. So as mobile phone show black page repeatedly when activating camera in processing the App Inventor program. Through something that research in library, web search and rich experiences programmer's explanation. Successfully to activated camera to recognize the QR code. TinyDB database is a difficult problem is when "Tag" mismatch in text box. After should be affected save and reload data at text box. Worse caused the system take up more memory and resources. I tried to use the transaction number as "Tag" in front of file name for separate to save each file accordingly. The Firedase is very easy development database storage system. However, against large data or complex user behaviours are very difficult to process and analysis. As considered cost/facility of preparing servers, operating systems, and database management systems are expensive. A free version of Firebase supports fewer than 100 connections at same time. A number of 100 network connections is satisfaction for majority of apps.

Finally, I have the honour with gratitude depot teams and station front line team's colleagues to support. During the trial test, mostly give us their comment/feedback. Accordance with their operating app's behaviours records. Let me a comprehensive modification of the interface or notification box optimisation.

The project was considered successful with my achieved the goal "Line Clear" with details as follow:

1. To reduce the incident relating red flashing light in track.

2. As safety, affective and reliable service can be provided through continual improvement of our systems. The approach to reducing incident relating red flashing light left in track may be extended to other systems in maintaining a safe environment to staff.

3. The project solution is result of the joint efforts in delivering better service to both internal and external customers.

\subsection{Recommendation}

Currently, frontline maintenance staff in normally provide with duty smartphone which is actually the required tool for installation of mobile apps. This phone can actually serve as a tool/reminder for reducing incidents. The previous trial proved that the mobile apps can effectively ensure the removal of protection arrangement upon completion of work. Thus, I should proceed with standardization in the aspects: 


\section{Implement on Corporation}

I would like to recommend using mobile apps to record the application/removal of red flashing light on all train service lines such that the completion of this critical task can be verified under relevant controllers.

\section{Extent the application}

If the process is proved to be effective, I would explore to extent its applications including to applying portable earth, safety padlock, barriers and possibly other important matters. Further improvement of the system can be considered in one possible direction is to develop a centralized database system to:

1. Works for single possession with multiple inputs, e.g. several work teams to prepare protections at a different location, possession granted.

2. Create an assignment by the system, team members to follow the assigned duty and confirm accordingly. The system will validate the correctness and completeness.

\section{Link with official system}

The work involving track access is normally recorded in official system. If such mobile application can link with official system. The record can only be finished when all protection arrangements have been removed. In other words, concerned risk can further be mitigated and almost eliminated.

\section{REFERENCES}

[1] Egoditor, QR Code Generator. Available from: Web site: https://www.qr-code-generator.com/ [Accessed: January 12, 2020].

[2] (https://appinventor.mit.edu/) Massachusetts Institute of Technology (2012), MIT App Inventor. Available from: Massachusetts Institute of Technology, Computer Science and Artificial Intelligence Laboratory Web site: https://appinventor.mit.edu/ [Accessed: February 2, 2020].

[3] Jason, Tyler (2011), App inventor for Android: build your own apps-- no experience required, Chichester, UK.

[4] 鄧文淵\&李淑玲 (2015), App inventor of database project, Gotop, Taipei, Taiwan.

[5] 鄧文淵\&李淑玲 (2014), App inventor of basic, Gotop, Taipei, Taiwan.

[6] Alpha Camp, What's Firebase. Available from: Web site: https://tw.alphacamp.co/blog/2016-0722-firebase [Accessed: January 10, 2020].

[7] Chih-hung Wu, Firebase.pptx. Available from: National TaiChung University of Education, Dept. of Digital Content and Technology Web site: http://120.108.221.55/profchwu/dctai/\%E6\%95\%99\%E6\%9D\%90/App\%20Inventor/Firebase\% E8\%B3\%87\%E6\%96\%99\%E5\%BA\%AB/firebase.pdf [Accessed: January 23, 2020].

[8] Google, Product. Available from: Web site: https://firebase.google.com/ [Accessed: December 24, 2019].

[9] Ben Sin (2019), First impressions of LG G8 and V50: 5G-ready and a second screen or infrared palm unlock. Available from: Web site: https://www.scmp.com/lifestyle/gadgets/article/2187684/first-impressions-lg-g8-and-v50-5gready-and-second-screen-or [Accessed: January 23, 2020].

[10] 蕭佑和 Youhesiao (2019), 只要 9 張圖, 看懂什麼是 5G. Available from: Web site: https://meethub.bnext.com.tw/\%E3\%80\%905g\%E7\%A7\%91\%E6\%99\%AE\%E3\%80\%91\%E5 \%8F\%AA\%E8\%A6\%819\%Е5\%BC\%B5\%Е5\%9C\%96\%ЕF\%BC\%8C\%Е7\%9C\%8B\%E6\%87 \%82\%E4\%BB\%80\%Е9\%BA\%BC\%E6\%98\%AF5g\%EF\%BD\%9C\%E5\%A4\%A7\%E5\%92\%8 C\%E6\%9C\%89\%E8\%A9\%B1\%E8\%AA\%AA/ [Accessed: January 7, 2020]. 
International Journal of Computer Science \& Information Technology (IJCSIT) Vol 12, No 4, August 2020

[11] 5G Infrastructure Public Private Partnership (5G PPP), "Business Validation in 5G PPP vertical use cases", 5G Infrastructure AssociationVision and Societal Challenges Working Group Business Validation, Models, and Ecosystems Sub-Group, <https://5g-ppp.eu/whitepapers/> [Accessed: June, 2020].

[12] $5 \mathrm{G} \quad \mathrm{CISCO}, \quad<\mathrm{https}: / / \mathrm{www} . c i s c o . c o m / c / d a m / g l o b a l / z h \_c n / s o l u t i o n s /$ serviceprovider/5g/pdf/index.html> [Accessed: July 2020].

\section{AUTHORS}

Tony Tsang (MIEEE'2000) received the BEng degree in Electronics \& Electrical Engineering with First Class Honours in U.K., in 1992. He studied the Master Degree in Computation from Computing Laboratory, Oxford University (U.K.) in 1995. He received the Ph.D from the La Trobe University (Australia) in 2000. He was awarded the La Trobe University Post-graduation Scholarship in 1998. He works in Hong Kong Polytechnic University as Lecturer since 2001. He works in Hong Kong College of Technology in 2014. He has numerous publications (more than 110 articles) in international journals and conferences and is a technical reviewer for several international journals and conferences. His research interests include mobile computing, networking, protocol engineering and formal methods. Dr. Tsang is a member of the IET and the IEEE.

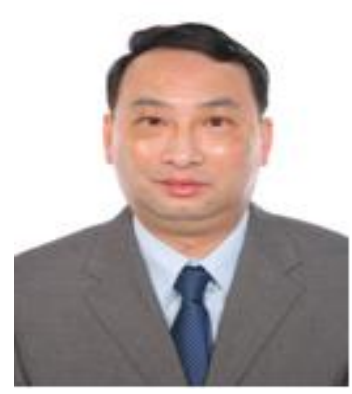

